\title{
Design and Simulation Analysis of Cam- type ATC Device
}

\author{
Zhenghao Ge, Jiawei Li and Peng Cao \\ School of Mechanical and Electrical Engineering, Shaanxi University of Science and Technology, Xi'an 710021, China
}

\begin{abstract}
The cam-type ATC device has the advantages of fast tooling and high-efficiency, but its structure is complicated and the design is more difficult. The main research contents of this paper is: carried out the cam three-dimensional modeling and the mechanism simulation analysis through the ATC device tool changer common program based on Creo and ADAMS. Established the ATC device assembly model and virtual prototype. Analyzed the ATC device tool change mechanism structure principle and the motion process. And determined the program's feasibility through the analysis results. At the same time, in the establishing dynamic simulation model process, studied the system and the component parameter setting, which is of reference value for the same type mechanism simulation.
\end{abstract}

Key words-ATC device; globoid cam simulation analysis; virtual prototyping

\section{INTRODUCTION}

ATC device as an important supporting parts of the processing center, its performance directly affects the development level of China's processing center. From the late 1950s, many countries began to study ATC devices. In 1958 the United States K \& T company developed with ATC device processing center. After 1978, CNC machine tools towards to enter the era of rapid development and achieve multi-process processing direction. In 1983, the International Organization for Standardization ISO developed a CNC tool holder with the international standard, then the automatic tooling system structure model has taken shape ${ }^{[1-2]}$, then in the 20 th century 70 years the rapid development of $\mathrm{CNC}$ machine tool industry into the golden age. In recent years, with the $\mathrm{CNC}$ machine tool processing center toward the multi-functional, high-speed and high reliability direction, the development of high load, high precision and high stability of the automatic tool changer has become an inevitable trend ${ }^{[3-4]}$. ATC cam-type automatic tool changer robot to be completed grasping knife, loosing knife, pulling knife, changing knife, slotting knife, clamping, reset 7 actions in a working cycle. The multi-ridge cam mechanism controls the completion of manipulator's grasping, changing, reset action, oscillating follower globoid cam mechanism controls the completion of pulling, slotting action. In this paper, the design and simulation analysis of cam mechanism in ATC device is carried out by using software such as CREO and ADAMS.

\section{AtC Device CAM Mechanism PRINCIPLE}

\section{A. ATC Device Cam Mechanism of the Common Program}

This article mainly introduces the double-globoid cam mechanism ATC device. The rotary motion of the device is achieved by means of a multi-ridge globoid cam mechanism, which oscillating follower globoid cam mechanism drive the fork to control the reciprocating linear motion of the arm. This ATC device change the spindle of the rotary motion into the output shaft of the reciprocating linear motion, to overcome the traditional swing mechanism transmission gap and the impact of large shocks. Its structure is shown in Fig. I:

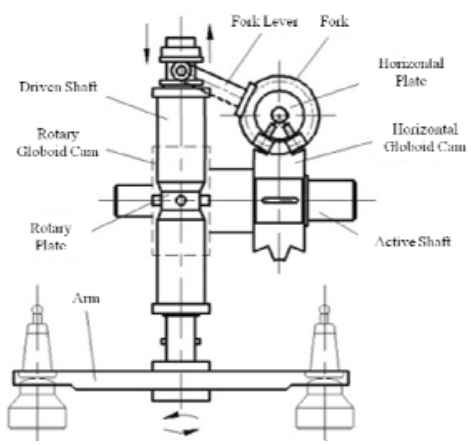

FIGURE I. DOUBLE-GLOBOID CAM-TYPE TOOL CHANGE MECHANISM

\section{B. ATC Device Tool Change Process}

According to the ATC device tooling work, design the motion cycle diagram by determining the initial rotation angle, the grasping angle, the reset angle of the multi-ridge cam, the pulling angle, the slotting angle, the loosing angle of, the clamping angle of oscillating follower globoid cam, Fig. II, III shows the motion cycle of the multi-ridge globoid cam and the oscillating follower globoid cam. Then the ATC device in a working cycle tool change process is as follows: globoid cam rotates $7.5^{\circ}$, the robot began to grasp knife; when the cam rotates from $7.5^{\circ}$ to $57.5^{\circ}$, arm rotates $65^{\circ}$ cutting tool; globoid cam rotates from $57.5^{\circ}$ to $72.5^{\circ}$, the arm loose knife; globoid cam rotates from $72.5^{\circ}$ to $137.5^{\circ}$, the arm straight move out of the tool; globoid cam from $117.5^{\circ}$ rotates to $242.5^{\circ}$, the arm rotates $180^{\circ}$ for tool changing; when the cam rotates from $222.5^{\circ}$ to $287.5^{\circ}$, the arm is inserted into a tool; when the cam rotates from $287.5^{\circ}$ to $302.5^{\circ}$, the arm clamps the tool; the cam rotates from $302.5^{\circ}$ to $352.5^{\circ}$, the arm rotates $65^{\circ}$ in the reverse direction, and returns to the initial position. The cam continues rotates by $7.5^{\circ}$ to complete a tool change process. 


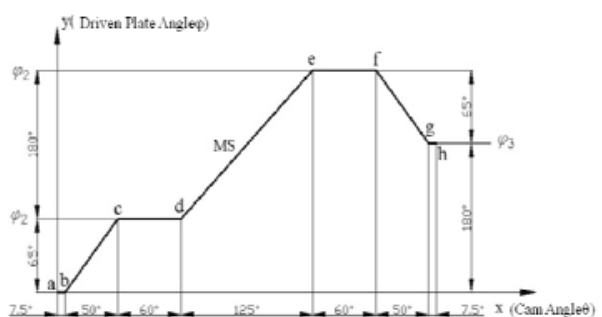

FIGURE II. THE MOVEMENT CYCLE OF MULTI-RIDGE GLOBOID CAM

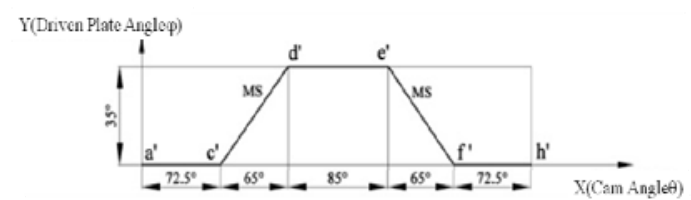

FIGURE III. THE MOVEMENT CYCLE OF OSCILLATING FOLLOWER GLOBOID CAM

\section{ATC DEVICE CAM MECHANISM DESIGN MODELING}

\section{A Design of Long Head Cam}

By the movement of multi-ridge globoid cam, the cam has two elevations of one return and four stop periods, which correspond to the loosing, pulling, slotting, clamping action, the first lift corresponds to the manipulator turn $65^{\circ}$, the second lift corresponds to the manipulator rotates $180^{\circ}$ change tool, the return corresponds to the robot reverse $65^{\circ}$ reset.

According to the working process and the motion cycle of the multi-ridge globoid cam, the CREO curve function can be used according to the theoretical profile equation of the globoid cam, the conjugate contact equation between the cam and the roller and the modified sinusoidal motion law to establish the different motion segments curve ${ }^{[5]}($ Fig. IV(a)), and mix these curve boundaries to produce the theoretical contour (as shown in Fig. IV (b)), finally normal bias ${ }^{[6]}$ the theoretical contours, resulting in the globoid cam solid model as shown in Fig. IV (c):
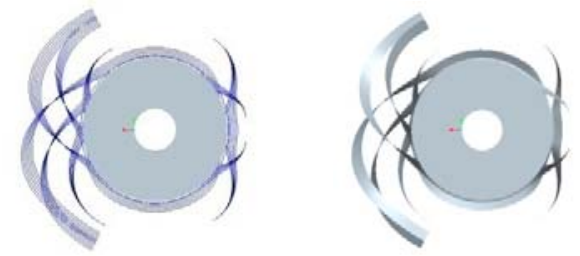

(a) globoid cam theory contour curve (b) globoid cam theory contour surface

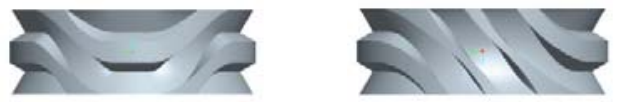

(C) globoid cam solid model

FIGURE IV. MODELING PROCESS OF MULTI-RIDGE GLOBOID CAM

\section{B Design and Modeling of the Oscillating Follower Globoid Cam}

In a working cycle of the ATC, the oscillating follower globoid cam mechanism controls the manipulator to complete the pulling and slotting two movements. The working cycle of the oscillating follower globoid cam is divided into five stages: stop, forward, stop, reverse and stop. Combined with the convex cam theory, the conjugate contact equation between the cam and the roller and the law of the modified sinusoidal motion, we can write the cam profile curve of the different stopping period and the moving section roller, put the pieces of program into the CREO generated oscillating follower globoid cam theory curve as shown in Fig. V (a), border mixture the curve, surface merging and physical generation . The final solid model is shown in Fig. V (b) (c):
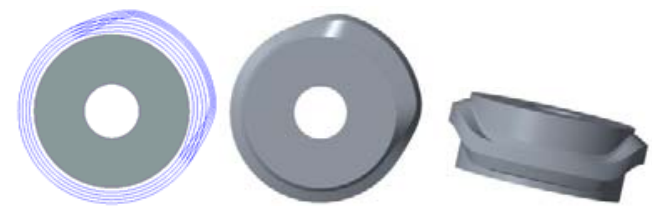

a) globoid cam theory contour curve b) globoid cam solid model c) globoid cam section profile surface

FIGURE V. MODELING PROCESS OF OSCILLATING FOLLOWER GLOBOID CAM

\section{ATC Device CAm Mechanism Movement AnAlysis}

\section{A. Movement Analysis of Long Head Cam Mechanism}

First define the simulation model ${ }^{[7]}$. Define material properties: Define the material of the organization as steel; Add gravity acceleration to the mechanism; Define the mechanism's simulation constraints, and then define the contact force of the mechanism. ADAMS usually gives two different ways to calculate the contact force between two objects, Impact and Restitution. Among them, the Impact model is an equivalent spring damping model, suitable for unilateral collision; The Restitution model is suitable for multiple collisions. According to the material of the cam and the index roller, the geometric parameters determine the Stiffness of the contact, the force exponent, the damping, the penetration depth ${ }^{[8]}$, this is the key to establishing a virtual prototyping of the cam mechanism. In order to reduce the calculation, usually in the modeling, the driven plate and roller built as one. The contact between the globoid cam and the driven plate can be equivalent to two variable radius cylinder collision problem, the following is ADAMS software for the definition of the collision force ${ }^{[9]}$ :

$$
F_{\text {_impact }}= \begin{cases}0 & q \geq q_{0} \text { where: } \\ K\left(q_{0}-q\right)^{e}-C \times(d q / d t) \times \operatorname{STEP}\left(q, q_{0}-d, 1, q_{0}, 0\right) & q<q_{0}\end{cases}
$$

STEP - step function;

$\mathrm{q}_{0}$ - the initial distance between two objects;

q-the actual distance of the object during the collision.

When $\mathrm{q}>\mathrm{q}_{0}$, the two objects will not touch, so the collision force is zero; when $\mathrm{q}<\mathrm{q}_{0}$, the two objects collide, the collision force relates to stiffness coefficient $K$, deformation $q-q_{0}$, force index value $\mathrm{e}$, the damping coefficient $\mathrm{C}$ and the deformation displacement $\mathrm{d}$ when the damping is fully applied.

Two-variable curvature of the cylinder collision contact 
normal force $\mathrm{P}$ and deformation $\delta$ relationship is:

$$
\mathrm{P}=\mathrm{K} \times \delta^{3 / 2}
$$

$\mathrm{K}$ and e value can be obtained. Since the change in force during the impact process is mainly controlled by the spring, the damping coefficient $\mathrm{C}$ is generally small and negligible.

The contact stiffness can be calculated using the following formula:

$$
\mathrm{K}=(4 / 3) \mathrm{R}^{1 / 2} \cdot \mathrm{E}^{*}
$$

Where $1 / \mathrm{R}=1 / \mathrm{R}_{1}+1 / \mathrm{R}_{2}, \mathrm{R}_{1}$ and $\mathrm{R}_{2}$ are the equivalent radius of the cam and roller contact points, respectively.

$1 / E^{*}=\left(1-\mu_{1}^{2}\right) / E_{1}+\left(1-\mu_{2}^{2}\right) / E_{2}, \mu_{1}, \mu_{2}$ are the Poisson's ratio of the cam and roller materials, $E_{1}$ and $E_{2}$ are the Young's modulus of the cam and roller material . Get the material from the inquiry manual:

$$
\mathrm{E}^{*}=1 /\left[\left(1-\mu_{1}^{2}\right) / \mathrm{E}_{1}+\left(1-\mu_{2}^{2}\right) / \mathrm{E}_{2}=1.13 \times 10^{5} \mathrm{~N} / \mathrm{mm}^{2}(3)\right.
$$

Ignore the influence of the roller radius on the curvature of the cam, so $1 / \mathrm{R} \approx 1 / \mathrm{R}_{2}, \mathrm{~K}=4.76 \times 10^{5} \mathrm{~N} / \mathrm{mm}^{2}$, $\mathrm{e}=1.13$

Finally add the drive, the establishment of the multi-ridge globoid cam digital prototypes shown in Figure 6:

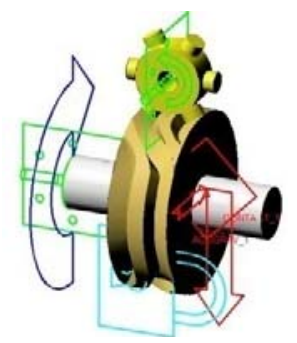

FIGURE VI. THE ENTITY MODEL OF MULTI-RIDGE GLOBOID CAM MECHANISM

\section{B Motion Analysis Results}

The simulation results are done by the postprocessing module PostProcessor, which can clearly view the simulation results in the form of animations and curves. In this paper, the angular displacement, angular velocity and angular acceleration of the driven plate geometric center are studied.

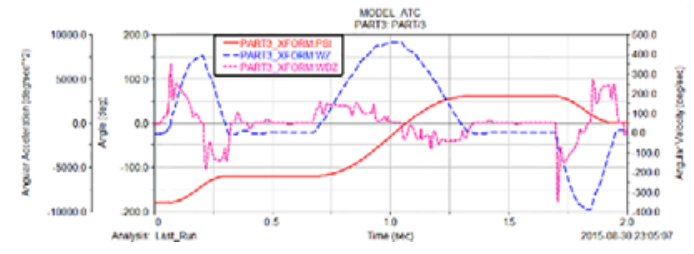

FIGURE VII. SIMULATION RESULTS OF LONG HEAD CAM MECHANISM

As shown in Fig. VII, the three curves represent the angular displacement, angular velocity, and angular acceleration curves of the driven plate. From the simulation results of the mechanism, it can be seen that the angular displacement curve of the multi-ridge cam mechanism is consistent with the theoretical angular displacement curve. The velocity peak appears in the segmentation section, which corresponds to the manipulator's grasping, changing, reset action. The degree acceleration of the driven plate is larger in the moving range, due to the sinusoidal motion cam itself has a cross-impact, and the curved surface of the cam is complex and connected by modeling into the surface is not smooth enough $^{[10]}$.

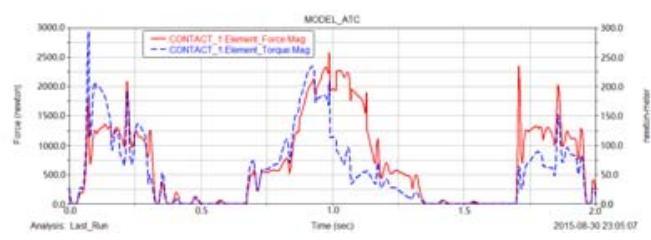

FIGURE VIII. COLLISION FORCE AND TORQUE OF MULTI-RIDGE GLOBOID CAM MECHANISM

As shown in Fig. VIII, the two curves represent the contact force and the contact torque curve between the driven plate and the cam. From the simulation results it can be seen that the contact force and the peak of the contact torque appears in the multi-ridge cam mechanism, the contact force and the contact moment are zero at the stop period. That verified the correctness of the model.

\section{Analysis of the Movement of the Cam Mechanism of the Swing Roller Follower}

The same method is used to analyze the motion of the oscillating follower globoid cam. As shown in Fig. IX, the three curves represent the displacement, velocity, and acceleration curves of the driven plate. From the simulation results of the mechanism, it can be seen that the angular displacement peak of the motion of the oscillating follower is $35^{\circ}$, the angle of the moving plate is consistent with the theoretical value. There are no jitter in the angular velocity curve. The acceleration curve of the driven plate is basically no jitter, the cam mechanism vibration is small.

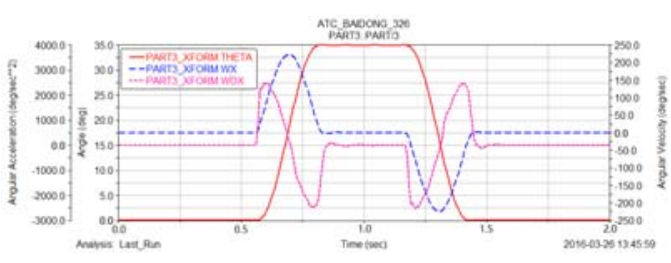

FIGURE IX. SIMULATION RESULTS OF THE CAMMING MECHANISM OF THE OSCILLATING ROLLER FOLLOWER

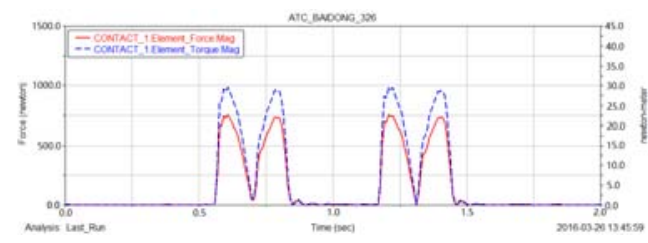

FIGURE X. COLLISION FORCE AND TORQUE OF OSCILLATING FOLLOWER GLOBOID CAM

As shown in Fig. $\mathrm{X}$ the two curves represent the contact force and contact torque curves between the driven plate and the cam. By comparing the contact force and the contact 
torque of the oscillating follower globoid cam mechanism, it can be found that the contact force and the contact torque of the multi-ridge cam mechanism is larger than those of the oscillating follower globoid cam mechanism, and the impact of the surface cam mechanism is smaller.

The establishment of its digital prototype as shown:

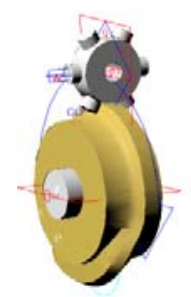

FIGURE XI. ESTABLISHMENT DIGITAL PROTOTYPE OF OSCILLATING FOLLOWER GLOBOID CAM MECHANISM

\section{ATC Device Assembly Model And The ESTABLISHMENT Of ViRTUAL PROTOTYPE}

Thus the establishment of ATC device assembly model and virtual prototype as shown:
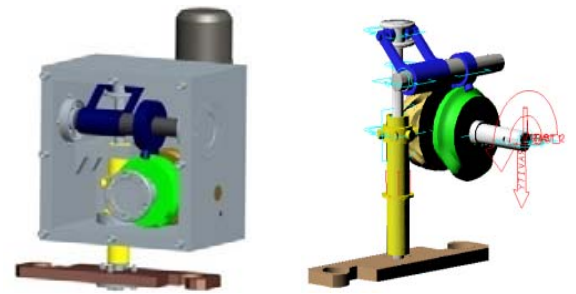

FIGURE XII. ASSEMBLY MODEL OF ATC DEVICE AND ESTABLISHMENT OF VIRTUAL PROTOTYPING

\section{Conclusions}

The cam type ATC device is generally used in the machining center, requiring the robot to move smoothly and reliably. Using the mechanical dynamics analysis software ADAMS analysis the virtual prototyping dynamic simulation and motion of the multi-ridge and the oscillating follower cam mechanism from CREO. Output angular displacement, angular velocity and angular acceleration, and verified the correctness of the single mechanism model is. In the process of establishing the dynamic simulation model, the parameter setting of the system and the component is studied, which is of reference value for the simulation of the same type of mechanism

\section{ACKNOWLEDGEMENTS}

This project is supported by Xi'an Science and Technology Project: CXY1431 (3)

Shaanxi Provincial Department of Education service local special projects: $15 \mathrm{JF} 010$

\section{REFERENCES}

[1] Li J M, Tao W J, Feng H T. Development status and key technology of automatic tool changer. Machine Tools and Hydraulic, 2013,(05):174-176+160.

[2] Li J Z. Research on improving the tool - making efficiency of machining center. Tool Technology, 2012, (02): 40-44.
[3] Zhong J H. Research on dynamic characteristics of globoid cam employed in automatic tool changer (ATC) of machine center. Applied Mechanics and Materials, 2013, (1) : 133-136.

[4] Yue M, Zhang S J, Jing Z. Study on the geometric characteristics of mating surfaces of globoid cam mechanisms. Mechanism and Machine Theory, 2016 (01) : 44-62.

[5] Zhang W G, Wang B Z, Gong Q Z. Solid modeling and processing simulation of multi-ridge globoid cam in ATC. Mechanical Design, 2015 , (05): 30-34

[6] Cao P, Ge Z H, Liu X Q. Three - dimensional modeling and motion simulation of multi-ridge globoid cam. Mechanical Transmission, 2016 (07) : 97-100.

[7] Yang D X, Zhang A H. Construction and analysis of virtual prototype of continuous indexed globoid cam mechanism based on ADAMS. Journal of Mechanical Industry Standardization and Quality, 2013, (08): 19-21.

[8] Ma Q C, Fang T. Dynamics simulation analysis of exchange cutter manipulator based on RecurDyn. Applied Mechanics and Materials, 2013, 364(2): 396-400.

[9] An X B, Pan S F. Contact collision model analysis in multi-body system dynamics simulation. Computer Simulation, 2008, (10): 98-101.

[10] Zhao Y X, Chang Z Y, Huang S G, Wang L. Dynamic simulation of cam indexing cam mechanism based on ADAMS. Microcomputer Information, 2010 (28): 1 